\title{
INVERSE SCATTERING FOR THE MATRIX SCHRÖDINGER OPERATOR AND SCHRÖDINGER OPERATOR ON GRAPHS WITH GENERAL SELF-ADJOINT BOUNDARY CONDITIONS
}

\author{
M. S. HARMER ${ }^{1}$
}

(Received 22 March, 2000)

\begin{abstract}
Using a parameterisation of general self-adjoint boundary conditions in terms of Lagrange planes we propose a scheme for factorising the matrix Schrödinger operator and hence construct a Darboux transformation, an interesting feature of which is that the matrix potential and boundary conditions are altered under the transformation. We present a solution of the inverse problem in the case of general boundary conditions using a Marchenko equation and discuss the specialisation to the case of a graph with trivial compact part, that is, with diagonal matrix potential.
\end{abstract}

\section{The matrix Schrödinger operator on the semi-axis}

We consider here the matrix Schrödinger operator on the semi-axis, that is,

$$
\mathscr{L} \equiv-\frac{d^{2}}{d x^{2}}+Q(x)
$$

on $L^{2}\left(\mathbb{R}_{+}, \mathbb{C}^{n}\right)$ where $\mathbb{R}_{+} \equiv[0, \infty)$. The potential matrix $Q(x)$ is assumed to be hermitian, absolutely integrable with absolutely integrable first moment and continuous on the open semi-axis [1]. The matrix Schrödinger operator, $\mathscr{L}_{0}$, defined on smooth functions vanishing at the origin and with compact support, is a symmetric operator with deficiency indices $(n, n)$. Using von Neumann extension theory [2] we may parameterise all self-adjoint extensions of this operator by unitary mappings between the deficiency subspaces, that is, $U \in \mathrm{U}(n)$. For practical purposes, however, it is more convenient to describe the self-adjoint extensions in terms of self-adjoint boundary conditions at the origin.

\footnotetext{
'Department of Mathematics, University of Auckland, New Zealand; e-mail: harmer@math.auckland.ac.nz.

(C) Australian Mathematical Society 2002, Serial-fee code 1446-1811/02
} 
It is well known that the construction of self-adjoint extensions is analogous to the description of Lagrange planes in a hermitian symplectic space [4]. In [4] these Lagrange planes are parameterised in terms of two $n \times n$ matrices such that their product is hermitian. Really, in the case of a hermitian symplectic space which admits a canonical basis, ${ }^{1}$ the Lagrange Grassmannian is isomorphic to the unitary group $\mathrm{U}(n)$ and we are able to explicitly parameterise the Lagrange planes, and hence selfadjoint boundary conditions, in terms of a unitary matrix - for details see [3]. In the case of the matrix Schrödinger operator on the semi-axis the self-adjoint boundary conditions at the origin are given by

$$
\left.\frac{i}{2}\left(U^{*}-\mathbb{a}\right) \psi\right|_{0}+\left.\frac{1}{2}\left(U^{*}+\mathbb{0}\right) \psi_{x}\right|_{0}=0 .
$$

Then the solution of the matrix Schrödinger equation $\mathscr{L} \Xi=\lambda \Xi$ with boundary values

$$
\left.\Xi\right|_{0}=\frac{1}{2}(U+\mathbb{0}) \equiv A,\left.\quad \Xi_{x}\right|_{0}=\frac{i}{2}(U-\mathbb{0}) \equiv B
$$

satisfies these boundary conditions. Using the Jost solutions, $F_{ \pm}$, solutions of the homogeneous equation $\mathscr{L} F_{ \pm}=\lambda F_{ \pm}$, with asymptotic behaviour

$$
\lim _{x \rightarrow \infty} F_{ \pm}(x, k) \sim e^{ \pm i k x} 0, \quad k=\sqrt{\lambda}
$$

and no prescribed behaviour at the origin, can be written as

$$
\Xi(x, k)=F_{-}(x, k) M_{-}(k)+F_{+}(x, k) M_{+}(k) .
$$

We define the scattering wave solution

$$
\Psi(x, k) \equiv \Xi(x, k) M_{-}^{-1}=F_{-}+F_{+} S(k),
$$

where $S(k)$ is known as the scattering matrix. The coefficients $M_{ \pm}$can be evaluated by taking the Wronskian of $\Xi$ and $F_{+}$or $F_{-}$[3]

$$
M_{ \pm}= \pm \frac{1}{2 i k}\left[\mathrm{~F}_{ \pm}^{\dagger} B-\mathrm{F}_{ \pm, x}^{\dagger} A\right],
$$

where $F_{ \pm}(k) \equiv F_{ \pm}(0, k)$ are known as the Jost functions and ${ }^{\dagger}$ is the involution $Y^{\dagger}(x, k) \equiv Y^{*}(x, \bar{k})$. The Wronskian of $\Xi^{\dagger}$ and $\Xi$

$$
W\left\{\Xi^{\dagger}, \Xi\right\}=\left.\left[\Xi^{\dagger} \Xi_{x}-\Xi_{x}^{\dagger} \Xi\right]\right|_{0}=A^{\star} B-B^{*} A=0,
$$

\footnotetext{
${ }^{1}$ By our definition, a hermitian symplectic space for a symmetric operator with non-equal deficiency indices does not admit a canonical basis or Lagrange planes [3].
} 
is always zero. Moreover, if we write $\Xi$ in terms of the scattering wave solutions

$$
\begin{aligned}
W\left\{\Xi^{\dagger}, \Xi\right\} & =M_{-}^{\dagger} W\left\{F_{-}^{\dagger}+S^{\dagger} F_{+}^{\dagger}, F_{-}+F_{+} S\right\} M_{-} \\
& =2 i k M_{-}^{\dagger}\left[-0+S^{\dagger} S\right] M_{-}=0
\end{aligned}
$$

we see, since $S^{\dagger}=S^{\star}$ for $k \in \mathbb{R}$, that the scattering matrix is unitary for real $k$.

If we diagonalise $U$, and use the well known asymptotics of the Jost functions $[1,3]$ in the above expression for $M_{ \pm}$, we see that the scattering matrix has the following asymptotic behaviour.

LEMMA 1.1. Given the self-adjoint operator $\mathscr{L}$, with associated unitary matrix $U$ defining the boundary conditions of $\mathscr{L}$, the scattering matrix of $\mathscr{L}$ has the asymptotics

$$
\lim _{k \rightarrow \infty} S(k) \sim \hat{U}
$$

where $\hat{U}$ is a unitary hermitian matrix $\hat{U}=\hat{U}^{\star}$ derived from $U$ by applying the map

$$
z \mapsto\left\{\begin{aligned}
1: z & \in \mathbb{J} \backslash\{-1\}, \\
-1: z & =-1
\end{aligned}\right.
$$

to the spectrum of $U$.

Here $\mathbb{T}$ is the unit circle in $\mathbb{C}$. The matrix $\hat{U}$, since it is hermitian, defines projections

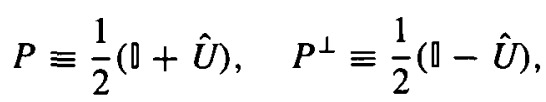

which may be used to define a factorisation of the original operator $\mathscr{L}$.

THEOREM 1.2. Given the self-adjoint operator $\mathscr{L}$ we can formally factorise it as

$$
\mathscr{L}=D^{\star} D
$$

where

$$
D=i\left[\frac{d}{d x}-V\right]
$$

and the functions in the domain of $D$ satisfy the following boundary conditions at the origin:

$$
\left.P^{\perp} \psi\right|_{0}=0 .
$$

Furthermore, $V$ is a hermitian matrix which satisfies the Riccati equation with the potential $Q(x)$ on the right-hand side and has initial value satisfying

$$
\left.P V\right|_{0}=-P H
$$

where $H$ is a bounded hermitian matrix specified by the boundary conditions of $\mathscr{L}$. 
PROOF. The fact that $V(x)$ satisfies the Riccati equation is well known. We diagonalise the matrix $U$. Suppose that $l$ of the eigenvalues of $U$ are -1 , then we choose the first $l$ elements of the basis used in the diagonalisation to be the eigenvectors with eigenvalue -1 . In this basis the boundary conditions of $\mathscr{L}$ can be written

$$
\left.\left[\begin{array}{cc}
-i 0_{(l)} & 0 \\
0 & -\tan (\bar{\varphi} / 2)
\end{array}\right] \psi\right|_{0}+\left.\left[\begin{array}{cc}
0 & 0 \\
0 & \mathbb{Q}_{(n-l)}
\end{array}\right] \psi_{x}\right|_{0}=0
$$

where $\psi$ is the boundary value (under the change of basis) and $\varphi$ is the $(n-l) \times(n-l)$ diagonal matrix with entries which are the eigenvalues of $U$ excluding the eigenvalues -1 . This means that we can write the boundary conditions for $\mathscr{L}$ as

$$
\begin{array}{r}
\left.P^{\perp} \psi\right|_{0}=0, \\
\left.P \psi_{x}\right|_{0}+\left.P H \psi\right|_{0}=0,
\end{array}
$$

where $H$ is the hermitian matrix depending on the original boundary conditions. Consider the operator $D^{*} D$. From $D$ we get the boundary condition

$$
\left.P^{\perp} \psi\right|_{0}=0
$$

and from $D^{*}$ we get the boundary condition

$$
\left.P[D \psi]\right|_{0}=0
$$

which is

$$
\left.P \psi_{x}\right|_{0}-\left.\left.P V\right|_{0} \psi\right|_{0}=0
$$

So we see that as long as the initial value of $V$ satisfies (5), $D^{*} D$ has the required boundary conditions.

It is well known that the Riccati equation can be linearised to the Schrödinger equation at zero energy; let us denote by $\Xi_{0}$ these zero-energy solutions. Then it is natural to ask how we can express the coefficient $V(x)$ in terms of $\Xi_{0}$. The following theorem is proved in [3], we merely quote it here.

THEOREM 1.3. The hermitian matrix $V$ can be written $V(x)=\Xi_{0, x}(x) \Xi_{0}^{-1}(x)$ where $\Xi_{0}(x)$ is the matrix of solutions of the Schrödinger equation at zero energy satisfying the boundary values of (2) specified by the unitary matrix $U_{0}$ and subject to:

(1) The matrix $U_{0}$ satisfies

$$
P U_{0}=P U
$$

(2) The potential $Q(x)$ is continuous in some neighbourhood containing the origin. 
The inverse problem for the matrix Schrödinger operator on the semi-axis with Dirichlet boundary conditions (that is, vanishing of the functions at the origin) is described in Agranovich and Marchenko [1]. In [3] the author extends Agranovich and Marchenko's result to arbitrary self-adjoint boundary conditions at the origin; the inverse problem may be reduced to a Marchenko equation ${ }^{2}$

$$
G(x+y)+K(x, y)+\int_{x}^{\infty} K(x, t) G(t+y) d t=0, \quad x<y,
$$

where

$$
G(t)=\sum_{l=1}^{N} C_{l}^{2} e^{-\kappa_{l} t}+\frac{1}{2 \pi} \int_{-\infty}^{\infty}(S(k)-\hat{U}) e^{i k t} d k .
$$

Here $\lambda_{l}=-\kappa_{l}^{2}$ are the discrete eigenvalues and the $C_{l}$ are non-negative hermitian matrices known as the normalisation matrices. The set $\left\{S(k) ; \kappa_{l}, C_{l}, l=1, \ldots, N\right\}$ is known as the scattering data. As this is a lengthy derivation we will not repeat it here. If we are able to solve (8) for the kernel of the transformation operator $K(x, y)$ we can recover the potential matrix from the well known identity [1]

$$
-2 \frac{d K(x, x)}{d x}=Q(x) .
$$

We can also recover the self-adjoint boundary conditions at the origin from the inverse problem via

$$
U=\left[\left.\Psi\right|_{0}-\left.i \Psi_{x}\right|_{0}\right]\left[\left.\Psi\right|_{0}+\left.i \Psi_{x}\right|_{0}\right]^{-1},
$$

which follows from the definition of the scattering wave solution plus (2). Consequently, the solution of the inverse problem allows us to recover not only the potential but also the self-adjoint boundary conditions at the origin.

\section{The Schrödinger operator on the graph with trivial compact part}

The motivation for studying the matrix Schrödinger operator is that, in the case of diagonal potential, it may be identified with the Schrödinger operator on the noncompact graph with trivial compact part-here we mean the graph consisting of $n$ semi-infinite rays with the origin of each ray identified with the single vertex of the graph. Although these are really two different operators, for the purposes of the inverse problem they may be identified: each component of the vector fuction on which the matrix Schrödinger operator acts is identified with the value of the function on one of the rays of the graph.

\footnotetext{
${ }^{2}$ It is also possible to reformulate it as a Riemann-Hilbert problem [3].
} 
In this case the self-adjoint boundary conditions at the origin play a crucial rôle; for instance if we have Neumann or Dirichlet ${ }^{3}$ boundary conditions there is no interaction between the rays and the graph decomposes into $n$ semi-axes for which the solution of the inverse problem is well known. The self-adjoint boundary conditions at the origin describe the interaction between the rays and they may also be thought of as inducing a 'zero-range' potential at the origin [3]. It is for these reasons that we consider the matrix problem with general boundary conditions above.

For the problem on the graph-the diagonal matrix potential-the matrix of Jost solutions is clearly a diagonal matrix and so too is the kernel $K(x, y)$. As a result the Marchenko equation (8) has along the diagonal $n$ scalar, independent (in the sense that each diagonal entry of $K(x, y)$ appears only once) Marchenko equations. Consequently, the inverse problem on the graph decouples to $n$ scalar inverse problems which can always be solved using only the $n$ diagonal entries of the scattering data ${ }^{4}$. Using this scattering data we can recover the potential on the rays plus the self-adjoint boundary conditions/zero-range potential at the origin.

If the self-adoint boundary conditions at the origin are specified it may be possible to recover the potential on the rays using a smaller set of scattering data. For Dirichlet or Neumann boundary conditions there is no interaction between the rays and we need all $n$ elements of the scattering data in order to recover the potential. On the other hand, if there is an even number of rays $n=2 m$ and we have boundary conditions at the origin so that the graph decomposes into $m$ copies of the whole real axis it is well known that we can recover the potential using only $m$ reflection coefficients and normalisation constants [5].

Let us consider flux-conserved boundary conditions which are defined by continuity at the origin

$$
\left.\phi_{1}\right|_{0}=\left.\phi_{2}\right|_{0}=\cdots=\left.\phi_{n}\right|_{0},\left.\quad \sum_{i=1}^{n} \phi_{i}^{\prime}\right|_{0}=0
$$

plus conservation of flux. These are self-adjoint and we provide a brief proof that in this case we need only $n-1$ of the diagonal elements of the scattering data in order to recover the potential (for details see [3]). We are able to show that the dispersion function is equal to [3]

$$
\operatorname{det}\left(2 i k M_{-}(k)\right) \equiv M(k)=\frac{i^{n-1}}{n} \mathfrak{f}_{+, 1} \mathfrak{f}_{+, 2} \ldots \mathfrak{f}_{+, n} \sum_{i=1}^{n} \frac{f_{+, i}^{\prime}}{f_{+, i}},
$$

\footnotetext{
${ }_{3}^{3}$ Agranovich and Marchenko [1] consider the inverse problem for the matrix operator on the semi-axis, however, they only consider Dirichlet boundary conditions which are not interesting in the case of diagonal potential.

${ }^{4}$ Actually, from (8) we need the diagonal entries of $S(k)$ and $C_{l}^{2}$.
} 
where $\mathrm{f}_{ \pm, i}$ denotes the Jost function for the $i$-th ray of the graph and $\mathrm{f}_{+, i}^{\prime}(k)=$ $\lim _{x \rightarrow 0} d f_{+, i}(x, k) / d x$. The scattering matrix has entries [3]

$$
S_{i j}(k)=\frac{2 i^{n} k \mathrm{f}_{+, 1} \mathrm{f}_{+, 2} \cdots \mathrm{f}_{+, n}}{n \mathrm{f}_{+, i} \mathfrak{f}_{+, j} M(k)}-\delta_{i j} \frac{\mathrm{f}_{-, i}}{\mathrm{f}_{+, i}} .
$$

Suppose we are given the first $n-1$ diagonal entries of the scattering matrix for real $k$, the reflection coefficients, $R_{j}(k) \equiv S_{j j}(k)$, plus the discrete eigenvalues $k_{l}=i \kappa_{l}$ and the first $n-1$ diagonal entries of the squares of the normalisation matrices, denoted $b_{l, j}$, for each eigenvalue. Since the Marchenko equation degenerates into $n$ independent scalar equations on the rays we can use this data to recover the potential and Jost solutions on the first $n-1$ rays. Equation (10) implies

$$
S_{i j}=\left[R_{i}+\frac{\mathrm{f}_{-, i}}{\mathrm{f}_{+, i}}\right] \frac{\mathrm{f}_{+, i}}{\mathrm{f}_{+, j}}, \quad \text { for } i \neq j .
$$

Consequently, we have enough information to recover the $(n-1) \times(n-1)$ minor of the scattering matrix formed by deleting the last column and row. Since $S(k)$ is unitary for $k \in \mathbb{R}$ we can recover the magnitudes of the remaining entries of the scattering matrix. Let us consider any entry in the last column not on the diagonal

$$
\left|S_{i n}\right|=\left|\frac{2 i^{n} k \mathrm{f}_{+, 1} \mathfrak{f}_{+, 2} \cdots \mathfrak{f}_{+, n-1}}{n \mathfrak{f}_{+, i} M(k)}\right|, \quad i \neq n .
$$

As we have the Jost functions for $i=1, \ldots, n-1$ we can solve this for the magnitude of the dispersion function on the real axis. Now $M(k)$ is analytic in the upper halfplane [3], has known magnitude on the real axis and known zeroes-the discrete eigenvalues - and so we can recover $M(k)$ in the upper half-plane. To do this we consider the 'normalised' dispersion function

$$
\hat{M}(k)=M(k) \frac{1}{i^{n}(k+i)} \prod_{l=1}^{N}\left[\frac{k+i \kappa_{l}}{k-i \kappa_{l}}\right]^{m_{l}} .
$$

Here $i \kappa_{l}$ are the zeroes and $m_{l}$ the orders of the zeroes of $M(k)$. In [3] we describe in detail how, using the $b_{l, i}$, to find the orders $m_{l}$ and also show there that, subject to the absence of vitual levels, $\hat{M}(k)$ is bounded and non-zero on the closed real axis with asymptotic

$$
\lim _{|k| \rightarrow \infty} \hat{M}(k)=1
$$

Furthermore, by definition $\hat{M}(k)$ is analytic in the upper half-plane with no zeroes there. By a simple application of the Cauchy integral formula and the Plemelje formula 
(see [5] for a similar calculation in the scalar case)

$$
\begin{aligned}
\arg M(k)= & \frac{1}{i}\left[\ln i^{n}(k+i)+\sum_{l=1}^{N} m_{l} \ln \frac{k-i \kappa_{l}}{k+i \kappa_{l}}\right]- \\
& -\frac{1}{\pi} \int_{-\infty}^{\infty} \frac{\ln \left[\left|M\left(k^{\prime}\right)\right| /\left|k^{\prime}+i\right|\right]}{k^{\prime}-k} d k^{\prime}
\end{aligned}
$$

where we take the principal value of the integral. Consequently we recover $M(k)$. From $M(k)$ we can use (10) to recover $S_{i n}$ for $i \neq n$. We now have enough information to recover the potential on the last ray: from the scattering matrix we can find $f_{+, n}$ and from $M(k)$ we can find $f_{+, n}^{\prime}$. These two functions are enough to recover the potential on the ray as they provide the scattering data for the Schrödinger operator on the semi-axis [1] (with Dirichlet boundary conditions in the cited text).

\section{Acknowledgements}

The author would like to thank Prof. B. S. Pavlov for his advice and many useful conversations.

\section{References}

[1] Z. S. Agranovich and V. A. Marchenko, The Inverse Problem of Scattering Theory (Gordon and Breach, New York, 1963).

[2] N. I. Akhiezer and I. M. Glazman, Theory of Linear Operators in Hilbert Space (Frederick Ungar Publishing, New York, 1966).

[3] M. S. Harmer, "The matrix Schrödinger operator and Schrödinger operator on graphs", Ph. D. Thesis, University of Auckland, 2000.

[4] V. Kostrykin and R. Schrader, "Kirchhoff's rule for quantum wires", J. Phys. A 32 (1999) 595-630.

[5] S. P. Novikov, S. V. Manakov, L. P. Pitaevskii and V. E. Zakharov, Theory of Solitons: The Inverse Scattering Method (Consultants Bureau, New York, 1984). 